\title{
PROPERTY $L$ AND ASYMPTOTIC ABELIANNESS FOR $W^{*}$-ALGEBRAS ${ }^{1}$
}

\author{
EDWARD SARIAN
}

\begin{abstract}
Let $Q$ be a finite $W^{*}$-algebra acting on a separable Hilbert space and having no abelian direct summand. If $\mathbb{Q}$ is asymptotically abelian, then $\mathbb{Q}$ has property $L$. (ii) Let $\mathbb{Q}$ be a finite $W^{*}$-algebra acting on a separable Hilbert space. Then $\mathbb{Q} \otimes B(h), h$ a separable infinite dimensional Hilbert space, is not asymptotically abelian. (iii) Type $\mathrm{II}_{\infty} W^{*}$-algebras are not asymptotically abelian. (iv) Noncommutative type I $W^{*}$-algebras are not asymptotically abelian. (v) The type III factor $\mathscr{B}=\mathscr{P} \otimes \mathbb{Q}(G)$ is not asymptotically abelian. $\mathscr{B}$ produces uncountably many nonisomorphic nonasymptotically abelian factors of type III and establishes an example of a purely infinite factor that has property $L$ but is not asymptotically abelian.
\end{abstract}

Let $Q$ be a $W^{*}$-algebra acting on a separable Hilbert space and let $\mathscr{Q}(\mathbb{Q})$ represent the set of unitary operators in $\mathbb{Q}$.

Definition 1. A uniformly bounded sequence $\left\{A_{n}\right\}$ in $\mathcal{Q}$ is called central if $\left\{A A_{n}-A_{n} A\right\} \rightarrow 0$ strongly for each $A \in \mathbb{Q} .\left\{A_{n}\right\}$ is called *-central if both $\left\{A_{n}\right\}$ and $\left\{A_{n}^{*}\right\}$ are central sequences.

In terms of central sequences, we define two forms of partial commutivity in $\mathbb{Q}$.

Definition 2. $Q$ has property $L$ if there is a central sequence $\left\{U_{n}\right\}$ contained in थ(Q) such that $\left\{U_{n}\right\} \rightarrow 0$ weakly.

Definition 3. $Q$ is called asymptotically abelian if there is a sequence of *-automorphisms $\left\{\phi_{n}\right\}$ on $\mathbb{Q}$ such that $\left\{\phi_{n}(A)\right\}$ is central for each $A \in \mathbb{Q}$.

Definition 2 is due to Pukánszky [4] and Definition 3 is essentially due to Sakai [5].

Recall that $Q^{\prime}$ denotes the commutant of $Q$ and is again a $W^{*}$-algebra. The center of $\mathcal{Q}$ is the abelian $W^{*}$-algebra defined by $\mathscr{Z}(\mathcal{Q})=\mathcal{Q} \cap \mathbb{Q}^{\prime}$. In [2] Dixmier defined a unique center valued function, denoted by $\#$, on a finite $W^{*}$-algebra that satisfies the following properties:

(a) if $\mathbb{Q} \in \mathscr{Z}(\mathbb{Q})$, then $A^{\#}=A$,

(b) $(\alpha A)^{\#}=\alpha A^{\#}$ for any scalar $\alpha$,

(c) $(A+B)^{\#}=A^{\#}+B^{\#}$,

(d) $(A B)^{\#}=(B A)^{\#}$,

(e) if $A \geqslant 0$, then $A^{\#} \geqslant 0$.

Received by the editors July 17, 1979 and, in revised form, September 25, 1979.

AMS (MOS) subject classifications (1970). Primary 46L10.

$K e y$ words and phrases. Property $L$, asymptotic abeliannness, finite $W^{*}$-algebra, type I $W^{*}$-algebra, type II $_{\infty} W^{*}$-algebra, type III factor, central trace, central sequence, *-automorphism.

${ }^{1}$ Portions of this paper are based on work that appears in the author's doctoral dissertation which was completed at Stevens Institute of Technology under the direction of Dr. Paul Willig. 
By [6, Theorem 2.4.6], \# is strongly and weakly continuous. We also observe for any *-automorphism $\phi$ on $\mathcal{Q},[\phi(A)]^{\#}=\phi\left(A^{\#}\right)$.

The following lemma will enable us to relate asymptotic abelianness to property $L$ in $W^{*}$-algebras of finite type. The author is indebted to his referee for improving Lemma 4 and providing the gist of its proof.

LEMMA 4. Let $\mathbb{Q}$ be a finite $W^{*}$-algebra acting on a separable Hilbert space and having no abelian direct summand. Then there exists $U \in \mathcal{Q}(\mathbb{Q})$ with $U^{\#}=0 .^{2}$

Proof. Assume first that $\mathcal{Q}$ is homogeneous of type $\mathrm{I}_{n}(1<n<\infty)$. Let $\left\{\boldsymbol{P}_{i}\right\}$ be a finite sequence of $n$ equivalent ${ }^{2}$ and mutually orthogonal projections in $Q$ with sum $I$. Let $\alpha$ be a primitive root of unity. Put $U=\sum_{i=1}^{n} \alpha^{i} P_{i}$. Then $U \in \mathcal{Q}(\mathbb{Q})$ and $U^{\#}=\left(\sum_{i=1}^{n} \alpha^{i}\right)(1 / n) I=0$. If $\mathbb{Q}$ is of type II, then there exists by [6, Proposition 2.2.13] a projection $P \in \mathbb{Q}$ equivalent to $I-P$. Then $P^{\#}=\left(\frac{1}{2}\right) I$. Put $U=I-$ $2 P$. Then $U \in \mathcal{Q}(\mathcal{Q})$ and $U^{\#}=0$. The general case follows by considering the appropriate direct sum decomposition of $\mathcal{Q}$, i.e., the required unitary is a direct sum of unitaries as specified in the present proof. Q.E.D.

THEOREM 5. Let $\mathbb{Q}$ be a finite $W^{*}$-algebra acting on a separable Hilbert space $h$ and having no abelian direct summand. If $\mathcal{Q}$ is asymptotically abelian, then $\mathbb{Q}$ has property $L$.

Proof. Let $\left\{\phi_{n}\right\}$ be a sequence of *-automorphisms making $Q$ asymptotically abelian. By Lemma 4, there exists $U \in \mathcal{Q}(\mathbb{Q})$ with $U^{\#}=0$. Define a central sequence of unitaries $\left\{V_{n}\right\}$ by $V_{n}=\phi_{n}(U)$. By weak compactness of the unit ball of $B(h),\left\{V_{n}\right\}$ has a subsequence $\left\{V_{k}\right\} \rightarrow V$ weakly. Since $\mathbb{Q}$ is weakly closed, $V \in \mathbb{Q}$. Since $\left\{V_{n}\right\}$ is central, $\left\{V_{k}\right\}$ is central and we need only show $V=0$. We have $\left\{A V_{k}-V_{k} A\right\} \rightarrow 0$ strongly and hence weakly for each $A \in \mathbb{Q}$. Also $\left\{A V_{k}-\right.$ $\left.V_{k} A\right\} \rightarrow A V-V A$ weakly for all $A \in \mathbb{Q}$. Thus $A V-V A=0$ or, equivalently, $V \in \mathbb{Q}^{\prime}$. Hence $V \in \mathscr{Z}(\mathbb{Q})$ and $V^{\#}=V$. By the remark following the stated properties of ${ }^{\#}, V_{k}^{\#}=\left[\phi_{k}(U)\right]^{\#}=\phi_{k}\left(U^{\#}\right)=0$ for each $k$. By the weak continuity of ${ }^{\#}, V^{\#}=0$. Thus $V=0$. Q.E.D.

The converse of Theorem 5 is false in the type II case. To see this, we need only consider the type $\mathrm{II}_{1}$ factor $\mathscr{Q}\left(\Pi \times \Phi_{2}\right)$. Here $\Pi$ denotes the group of those permutations of positive integers which leave all but finitely many integers fixed and $\Phi_{2}$ denotes the free group on two generators. $\mathcal{Q}\left(\Pi \times \Phi_{2}\right)$ is generated by the right translation operators on $L_{2}\left(\Pi \times \Phi_{2}\right)$ and has property $L$ (cf. [1, pp. 4-5] and [9]) but is not asymptotically abelian (cf. [1, Proposition I.1.24]).

We also know from [7] that type I $W^{*}$-algebras do not have property $L$. Hence by applying the contrapositive of Theorem 5 we can conclude the following corollary.

COROLlARY 6. Finite type I $W^{*}$-algebras having no abelian direct summand are not asymptotically abelian.

${ }^{2} E \sim F(E$ is equivalent to $F)$ iff there is a partial isometry $P \in \mathbb{Q}$ such that $P P^{*}=E$ and $P^{*} P=F$. 
We shall use Corollary 6 along with Theorem 8 to prove noncommutative type I $W^{*}$-algebras are not asymptotically abelian. Theorem 8 will enable us to conclude that $W^{*}$-algebras of types $\mathrm{I}_{\infty}$ and $\mathrm{II}_{\infty}$ are not asymptotically abelian. The following lemma will be needed.

LeMMA 7. Let $\mathscr{Q}$ be a properly infinite $W^{*}$-algebra. Then there is a nonzero projection $E \in \mathbb{Q}$ satisfying $E \sim I \sim I-E$.

Proof. By [6, Proposition 2.2.4] there exists a sequence $\left\{P_{n}\right\}$ of mutually orthogonal, equivalent projections in $\mathbb{Q}$ with $P_{n} \sim I$ and $I=\sum_{n=1}^{\infty} P_{n}$. Then by the remarks following [6, Definition 2.1.1],

$$
\sum_{n=1}^{\infty} P_{n} \sim \sum_{n=2}^{\infty} P_{n},
$$

so

$$
P_{1} \sim I \sim I-P_{1} \text {. Q.E.D. }
$$

THEOREM 8. Let $\mathbb{Q}$ be a finite $W^{*}$-algebra acting on a separable Hilbert space $k$. Then $\mathbb{Q} \otimes B(h), h$ a separable infinite dimensional Hilbert space, is not asymptotically abelian.

Proof. Suppose $\mathbb{Q} \otimes B(h)$ is asymptotically abelian. Then there exists a sequence of *automorphisms $\left\{\rho_{n}\right\}$ such that $\left\{\rho_{n}(T)\right\}$ is central for all $T \in \mathbb{Q} \otimes$ $B(h)$. Let $A, B \in \mathbb{Q} \otimes B(h)$. Then $\left\{A_{n}=\rho_{n}(A)\right\}$ and $\left\{B_{n}=\rho_{n}(B)\right\}$ are *-central sequences. From [3, Lemmas 4.2 and 4.3], we can find *-central sequences $\left\{F_{n}\right\}$ and $\left\{G_{n}\right\}$ in $Q \otimes\{I\}$ such that $\left\{A_{n}\right\},\left\{B_{n}\right\},\left\{A_{n}^{*}\right\}$ and $\left\{B_{n}^{*}\right\}$ are, respectively, equivalent to $\left\{F_{n}\right\},\left\{G_{n}\right\},\left\{F_{n}^{*}\right\}$ and $\left\{G_{n}^{*}\right\}$. This means $\left\{A_{n}-F_{n}\right\},\left\{B_{n}-G_{n}\right\}$, $\left\{A_{n}^{*}-F_{n}^{*}\right\}$ and $\left\{B_{n}^{*}-G_{n}^{*}\right\}$ each converge strongly to zero. Write $A_{n} B_{n}-B_{n} A_{n}=$ $\left(A_{n}-F_{n}\right) B_{n}+F_{n}\left(B_{n}-G_{n}\right)$. Since $\left\{B_{n}\right\}$ is bounded and $\left\{A_{n}^{*}-F_{n}^{*}\right\}$ converges strongly to zero, we have that $\left\{\left(A_{n}-F_{n}\right) B_{n}\right\}$ converges weakly to zero. Similarly, the boundedness of $\left\{F_{n}\right\}$ and strong convergence of $\left\{B_{n}-G_{n}\right\}$ imply that $\left\{F_{n}\left(B_{n}\right.\right.$ $\left.\left.-G_{n}\right)\right\}$ converges weakly to zero. Thus $\left\{A_{n} B_{n}-F_{n} G_{n}\right\} \rightarrow 0$ weakly and the same reasoning will show that $\left\{B_{n} A_{n}-G_{n} F_{n}\right\} \rightarrow 0$ weakly also.

Now let $\psi$ be a normal state on $B(h)$ and $\tau$ a normal tracial state on $\mathbb{Q}$ (cf. [6, p. 90]). Then $\theta=\tau \otimes \psi$ is a normal state on $Q \otimes B(h)$ and since $\theta$ coincides with $\tau$ when restricted to $\mathbb{Q} \otimes\{I\}$, we have $\theta\left(F_{n} G_{n}\right)=\theta\left(G_{n} F_{n}\right)$. Also $\theta\left(A_{n} B_{n}-F_{n} G_{n}\right) \rightarrow$ 0 and $\theta\left(B_{n} A_{n}-G_{n} F_{n}\right) \rightarrow 0(n \rightarrow \infty)$ since $\theta$ is weakly continuous on bounded sets. It follows readily that $\theta\left(A_{n} B_{n}-B_{n} A_{n}\right) \rightarrow 0(n \rightarrow \infty)$.

Define a sequence $\left\{\phi_{n}\right\}$ of normal states on $\mathbb{Q} \otimes B(h)$ by $\phi_{n}(T)=\theta\left(\rho_{n}(T)\right)$. Then by weak-* compactness of bounded sets in the dual, $\left\{\phi_{n}\right\}$ has an accumulation point $\phi$ and a subsequence $\left\{\phi_{k}\right\}$ such that $\phi_{k}(A B-B A) \rightarrow \phi(A B-B A)$ $(k \rightarrow \infty)$. Also $\phi_{k}(A B-B A)=\theta\left(\rho_{k}(A B-B A)\right)=\theta\left(A_{k} B_{k}-B_{k} A_{k}\right) \rightarrow 0(k \rightarrow \infty)$ so that $\phi(A B-B A)=0$. Thus $\phi$ is a state on $Q \otimes B(h)$ that satisfies $\phi(A B)=$ $\phi(B A)$ for all $A, B \in \mathbb{Q} \otimes B(h)$.

By Lemma 7, there exists a nonzero projection $E \in Q \otimes B(h)$ such that $E \sim I$ $\sim I-E$. Let $P$ and $Q$ be partial isometries in $Q \otimes B(h)$ such that $P P^{*}=E$, $Q Q^{*}=I-E$ and $P^{*} P=I=Q^{*} Q$. Then $\phi(E)=\phi\left(P P^{*}\right)=\phi\left(P^{*} P\right)=\phi(I)=1$ 
and $\phi(I-E)=\phi\left(Q Q^{*}\right)=\phi\left(Q^{*} Q\right)=\phi(I)=1$. Also $\phi(I)=\phi(I-E)+\phi(E)=$ 2, a contradiction. Q.E.D.

The technique for proving Theorem 8 is essentially that of Theorem 4.4 in [3].

THEOREM 9. Type $\mathrm{II}_{\infty} W^{*}$-algebras are not asymptotically abelian.

Proof. Let $\mathcal{Q}$ be a $W^{*}$-algebra of type $\mathrm{II}_{\infty}$. By [10, Theorem 9], $\mathcal{Q}$ is spatially isomorphic to $\mathscr{B} \otimes B(h)$, where $h$ is a separable infinite dimensional Hilbert space and $\mathscr{B}$ is a type $\mathrm{II}_{1} W^{*}$-algebra. Then if $\mathscr{Q}$ is asymptotically abelian, and since strong convergence is preserved under *-isomorphism (cf. [6, Corollary 4.1.23]), $\mathscr{B} \otimes B(h)$ would be asymptotically abelian also. This contradicts Theorem 8 . Q.E.D.

THEOREM 10. Type $\mathrm{I}_{\infty} W^{*}$-algebras are not asymptotically abelian.

Proof. Let $\mathscr{Q}$ be a type $I_{\infty} W^{*}$-algebra. By [6, Theorem 2.3.3], $\mathscr{Q}$ is *-isomorphic to $\mathscr{Z}(\mathbb{Q}) \otimes B(h)$, where $h$ is a separable infinite dimensional Hilbert space. Then if $\mathbb{Q}$ is asymptotically abelian, $\mathscr{Z}(\mathbb{Q}) \otimes B(h)$ would be asymptotically abelian also. This contradicts Theorem 8. Q.E.D.

ThEOREM 11. Noncommutative type I $W^{*}$-algebras are not asymptotically abelian.

Proof. Let $Q$ be a noncommutative type I $W^{*}$-algebra. By [6, Theorem 2.3.2], $\mathbb{Q}$ can be decomposed uniquely into a direct sum of type $I_{n} W^{*}$-algebras $\mathbb{Q}_{n}(n \in K)$, where $K$ is a family of mutually distinct cardinal numbers. Now suppose $Q$ is asymptotically abelian and $\left\{\phi_{j}\right\}$ is a sequence of *-automorphisms on $\mathbb{Q}$ demonstrating the property. Write each $\phi_{j}$ as a direct sum whose $i$ th summand $\phi_{j i}$ is the restriction of $\phi_{j}$ to $\mathbb{Q}_{n_{i}}$. Each $\mathbb{Q}_{n_{i}}$ is homogeneous of degree $n_{i}$ (cf. [6, Definition 2.3.1]). Consequently, for each $j, \phi_{j i}$ is a ${ }^{*}$-automorphism on $Q_{n_{i}}$. Thus for each $i$, $\left\{\phi_{j i}\right\}$ is a sequence of *-automorphisms making $\mathbb{Q}_{n_{i}}$ asymptotically abelian. This contradicts Theorem 10 or Corollary 6 , since if $\mathscr{Q}$ is noncommutative, $K$ must contain a cardinal number $n$ satisfying $1<n<\infty$. Q.E.D.

We now turn our attention to factors of type III. It is not known whether there are any type III asymptotically abelian factors. It is natural to ask, however, if asymptotic abelianness implies property $L$ for purely infinite factors. We do not answer this question, but present for convenience a similar result proved by Glaser in [3] for the stronger property of asymptotic abelianness with invariant state and then prove by example that the converse is false. Our result also establishes a continuum of nonisomorphic nonasymptotically abelian factors of type III.

Definition 12 . Let $\mathcal{Q}$ be a type III $W^{*}$-algebra. $\mathcal{Q}$ is said to be asymptotically abelian with invariant state if there is a sequence of *automorphisms $\left\{\rho_{n}\right\}$ on $Q$ and a normal state $\phi$ on $Q$ satisfying the following conditions:

(i) $\left\{\rho_{n}(A)\right\}$ is central for each $A \in \mathbb{Q}$,

(ii) $\phi\left(\rho_{n}(A)\right)=\phi(A)$ for each $A \in \mathbb{Q}$ and each $n$.

THEOREM 13. Let $\mathbb{Q}$ be a type III factor acting on a separable Hilbert space. If $\mathbb{Q}$ is asymptotically abelian with invariant state then $\mathbb{Q}$ has property $L$. 
Proof. See [3, Proposition 3.4].

Theorem 14. Let $\mathcal{P}$ denote the Pukánszky type III factor and let $\mathscr{Q}(G)$ denote the type $\mathrm{II}_{1}$ factor generated by the right translation operators on $L_{2}(G)$, where $G$ is a discrete countable group whose conjugacy classes $C_{\mathrm{g}}=\left\{b g b^{-1} \mid b \in G, g\right.$ a fixed element in $G$ \} except $C_{e}$ are infinite ( $e$ is the identity in $G$ ). Then $\mathscr{P} \otimes \mathbb{Q}(G)$ is a type III factor that is not asymptotically abelian.

Proof. $\mathscr{P} \otimes \mathbb{Q}(G)$ is a type III factor by [6, Theorem 2.6.4]. Hence there is a nonzero projection $E \in \mathscr{P} \otimes \mathbb{Q}(G)$ satisfying $E \sim I \sim I-E$. Let $\tau$ be the trace in $\mathscr{Q}(G)$ and assume $\mathscr{P} \otimes \mathbb{Q}(G)$ is asymptotically abelian. Then there exists a sequence of ${ }^{*}$-automorphisms $\left\{\rho_{n}\right\}$ such that $\left\{\rho_{n}(T)\right\}$ is central for all $T \in \mathscr{P} \otimes$ $\mathcal{Q}(G)$. Let $A, B \in \mathcal{P} \otimes \mathbb{Q}(G)$. Then $\left\{A_{n}=\rho_{n}(A)\right\}$ and $\left\{B_{n}=\rho_{n}(B)\right\}$ are *-central sequences. From [6, Lemma 4.3.22] we can find *-central sequences $\left\{F_{n}\right\}$ and $\left\{G_{n}\right\}$ in $\{I\} \otimes \mathbb{Q}(G)$ such that $\left\{A_{n}\right\},\left\{B_{n}\right\},\left\{A_{n}^{*}\right\}$ and $\left\{B_{n}^{*}\right\}$ are, respectively, equivalent to $\left\{F_{n}\right\},\left\{G_{n}\right\},\left\{F_{n}^{*}\right\}$ and $\left\{G_{n}^{*}\right\}$. Then by applying the argument of Theorem 8 there exists a state $\phi$ on $\mathscr{P} \otimes \mathbb{Q}(G)$ with $\phi(I)=1$ and $\phi(I)=2$. Q.E.D.

COROllary 15. $P \otimes \mathbb{P}\left(\Pi \times \Phi_{2}\right)$ has property $L$ but is not asymptotically abelian.

Proof. $\mathscr{C}\left(\Pi \times \Phi_{2}\right)$ has $L . \mathscr{P} \otimes \mathbb{Q}\left(\Pi \times \Phi_{2}\right)$ has $L$ by [8, Theorem 2] and is not asymptotically abelian by Theorem 14 . Q.E.D.

COROLlaRY 16. There exists an uncountable family of nonisomorphic nonasymptotically abelian factors of type III on a separable Hilbert space.

Proof. This is an immediate consequence of Theorem 14 and [6, Corollary 4.3.24]. Q.E.D.

\section{REFERENCES}

1. S. Anastasio and P. Willig, The structure of factors, Algorithmics Press, New York, 1974.

2. J. Dixmier, Lex anneaux d'opérateurs de class finie, Ann. Sci. École Norm. Sup. (3) 66 (1949), 209-261.

3. M. Glaser, Asymptotic abelianness of infinite factors, Trans. Amer. Math. Soc. 178 (1973), 41-56.

4. L. Pukánszky, Some examples of factors, Publ Math. Debrecen. 4 (1956), 135-156.

5. S. Sakai, Asymptotically abelian $\mathrm{II}_{1}$-factors, Publ. Res. Inst. Math. Sci. Ser. A 4 (1968/69), 299-307.

6. __ $C^{*}$-algebras and $W^{*}$-algebras, Springer-Verlag, Berlin and New York, 1971.

7. E. Sarian, Property $L$ and $W^{*}$-algebras of type I, Tôhoku Math. J. 31 (1979), 491-494.

8. J. Tomiyama, On the tensor products of von Neumann algebras, Pacific J. Math. 30 (1969), 263-270.

9. P. Willig, Properties $\Gamma$ and $L$ for type $\mathrm{II}_{1}$ factors, Proc. Amer. Math. Soc. 25 (1970), 836-837.

10. __ Property $L$ and direct integral decompositions of $W^{*}$-algebras, Proc. Amer. Math. Soc. 31 (1971), 87-91.

Department of Computer and Information Science, New Jersey InstTtute of Technology, NeWARK, NeW JERSEY 07102 